\title{
Evidence for a Long-Lasting Compulsive Alcohol Seeking Phenotype in Rats
}

\author{
Chiara Giuliano ${ }^{*, 1,5}$, Yolanda Peña-Oliver ${ }^{1,5}$, Charles R Goodlett ${ }^{2}$, Rudolf N Cardinal ${ }^{3}$, Trevor W Robbins', \\ Edward T Bullmore ${ }^{1,3,4}$, David Belin ${ }^{1,6}$ and Barry J Everitt ${ }^{1,6}$ \\ 'Behavioral and Clinical Neuroscience Institute, Department of Psychology, University of Cambridge, Cambridge, UK; '² Department of Psychology, \\ Indiana University-Purdue University Indianapolis, Indianapolis, IN, USA; ${ }^{3}$ Department of Psychiatry, University of Cambridge, Cambridge, UK; \\ ${ }^{4}$ Clinical Unit Cambridge and Academic DPU, GlaxoSmithKline R\&D, Clinical Unit Cambridge, Addenbrooke's Hospital, Cambridge, UK
}

\begin{abstract}
Excessive drinking to intoxication is the major behavioral characteristic of those addicted to alcohol but it is not the only one. Indeed, individuals addicted to alcohol also crave alcoholic beverages and spend time and put much effort into compulsively seeking alcohol, before eventually drinking large amounts. Unlike this excessive drinking, for which treatments exist, compulsive alcohol seeking is therefore another key feature of the persistence of alcohol addiction since it leads to relapse and for which there are few effective treatments. Here we provide novel evidence for the existence in rats of an individual vulnerability to switch from controlled to compulsive, punishmentresistant alcohol seeking. Alcohol-preferring rats given access to alcohol under an intermittent 2-bottle choice procedure to establish their alcohol-preferring phenotype were subsequently trained instrumentally to seek and take alcohol on a chained schedule of reinforcement. When stable seeking-taking performance had been established, completion of cycles of seeking responses resulted unpredictably either in punishment ( $0.45 \mathrm{~mA}$ foot-shock) or the opportunity to make a taking response for access to alcohol. Compulsive alcohol seeking, maintained in the face of the risk of punishment, emerged in only a subset of rats with a predisposition to prefer and drink alcohol, and was maintained for almost a year. We show further that a selective and potent $\mu$-opioid receptor antagonist (GSKI52I498) reduced both alcohol seeking and alcohol intake in compulsive and non-compulsive rats, indicating its therapeutic potential to promote abstinence and prevent relapse in individuals addicted to alcohol.
\end{abstract}

Neuropsychopharmacology (2018) 43, 728-738; doi:I0.1038/npp.2017.105; published online 28 June 2017

\section{INTRODUCTION}

Alcohol addiction is characterized both by the inability to control drinking and the emergence of alcohol seeking habits, which can be characterized as compulsive because the behavior persists despite adverse consequences (American Psychiatric Association, 2013; Corbit et al, 2012; Everitt and Robbins, 2015; Koob and Volkow, 2010; McKim et al, 2016). Although the few treatments currently available all aim to reduce alcohol intake, none have been designed to reduce the compulsive seeking of alcohol that leads to drinking at relapse. In order to identify new effective therapeutic strategies, a better understanding of the psychological mechanisms that maintain alcohol seeking behavior in the face of adverse consequences would have great utility. These have hitherto been underexplored compared to the focus on drinking and associated intoxication.

*Correspondence: Dr C Giuliano, Behavioral and Clinical Neuroscience Institute, Department of Psychology, University of Cambridge, Downing Street, Cambridge CB2 3EB, UK, Tel: +44 01223 765292, Fax: +44 0 I 223 333564, E-mail: cg45 I@cam.ac.uk

${ }^{5}$ These authors contributed equally to this work.

${ }^{6}$ Co-last authors.

Received I8 March 2017; revised 9 May 2017; accepted I4 May 2017; accepted article preview online 29 May 2017
If the next generation of treatments for alcohol addiction are to be more effective than those currently available, a paradigm shift may be necessary in order to target also the psychological and neurochemical mechanisms underlying compulsive alcohol seeking that leads to relapse in vulnerable individuals (Spanagel, 2000), rather than targeting reductions in the amount of alcohol consumed when individuals already have relapsed, important though that is. This warrants the operationalization of compulsive alcohol seeking, as opposed to drinking, in translational animal models.

Many current procedures focus on measuring excessive, or inflexible alcohol drinking (reviewed in depth by Carnicella et al, 2014; Hopf and Lesscher, 2014), measured as the persistence of intake despite overt pairing with aversive consequences, including the punishment of instrumental responding under low fixed ratio (FR) schedules of reinforcement (Seif et al, 2013) or adulteration of the taste of alcohol (Marchant et al, 2013; Turyabahika-Thyen and Wolffgramm, 2006; Vengeliene et al, 2009; Wolffgramm, 1991), but they do not directly address the compulsive nature of alcohol seeking, which occurs prior to drinking and is mechanistically dissociable from the acute intoxicating effects of the drug. Therefore, based on our previous work in which we specifically investigated compulsive cocaine 
seeking behavior, demonstrating the utility of spatially and temporally separating seeking from taking instrumental responses (Pelloux et al, 2007), we have here developed a novel behavioral approach in rats to investigate the individual vulnerability to switch from controlled to compulsive alcohol seeking behavior. The interaction between the degree of exposure to drugs and vulnerability in exposed individuals has been shown to be critical in the development of drug addiction (Cooper et al, 2007; DerocheGamonet et al, 2004; Pelloux et al, 2007). The behavioral procedure we have developed will allow investigation of the neural basis of compulsive alcohol seeking while also offering a new, readily accessible tool, with high predictive value, for drug discovery.

People addicted to alcohol may persist for months, or even years, in compulsively seeking alcohol and remain vulnerable to relapse after even long periods of abstinence. Some treatments in clinical use, such as nalmefene or naltrexone, target $\mu$-opiate receptors and reduce volumes of alcohol drunk thereby reducing harm, or relapse rates in some individuals addicted to alcohol, respectively (Vendruscolo et al, 2012; Vengeliene et al, 2009; Vengeliene et al, 2014). However, while clinically significant these treatments are not optimal (Franck and Jayaram-Lindström, 2013; Hendershot et al, 2016; Rösner et al, 2010). Opioid receptor-mediated mechanisms have been shown to reduce alcohol drinking, craving for alcohol and established CS-controlled alcohol seeking (Vengeliene et al, 2008); moreover $\mu$-opioid receptors in the prefrontal context have been implicated in compulsive eating (Blasio et al, 2014). We have therefore investigated $\mu$-opioid mechanisms in compulsive alcohol seeking in our newly established procedure using the most selective and well-characterized, in vitro and in vivo (Giuliano et al, 2012, 2013; Ignar et al, 2011; Kelly et al, 2015; Ripley et al, 2015; Ziauddeen et al, 2013, 2016), $\mu$-opioid receptor antagonist GSK1521498. We have shown previously that this compound is more effective than naltrexone in reducing cue-controlled alcohol seeking and also alcohol drinking (Giuliano et al, 2015). These earlier data taken together with the present results provide substantial evidence for the potential efficacy of this full $\mu$-opioid receptor antagonist in the clinical treatment for alcohol addiction, since it is able to reduce compulsive alcohol seeking, drinking and the propensity to relapse induced by alcohol cue exposure.

\section{MATERIALS AND METHODS}

\section{Animals}

Male alcohol-preferring (P) rats ( $\sim 30$ days old, $n=61)$ were obtained from Indiana University Medical Center (Indiana, USA). See SOM. Experiments were conducted in accordance with the UK (1986) Animal (Scientific Procedures) Act (Project license 80/2234) and the GSK Policy on the Care, Welfare and Treatment of Laboratory Animals.

\section{Apparatus}

See SOM.
Assessment of the Drinking Phenotype in P Rats: Two-Bottle Choice Procedure

See SOM.

\section{Procedures}

The full series of experiments conducted is schematically summarized in Figure 1.

\section{Experiment 1: Establishment of Compulsive Alcohol Seeking Behavior}

(i) Compulsive alcohol seeking. The training consisted of the following 5 phases (see Figure 1a).

i. Pavlovian conditioning: see SOM.

ii. Taking phase: each cycle began with the insertion of the randomly assigned taking lever. Rats were trained to press the taking lever under a fixed-ratio 1 schedule of reinforcement, which resulted in $5 \mathrm{~s}$ CS illumination, extinction of the house light and delivery of $0.1 \mathrm{ml} \mathrm{15 \%}$ EtOH. Rats were limited to a maximum of 45 rewards/ 2 h-session.

iii. Seeking-taking phase: each cycle in the seeking-taking chained schedule of reinforcement began with insertion of the seeking lever (opposite the taking lever that was retracted). Seeking lever presses under a random interval (RI) schedule were never directly reinforced, but instead resulted in the extension of the taking lever and the simultaneous retraction of the seeking lever. Taking lever responses under FR1 resulted in the illumination of the stimulus light above the taking lever for $5 \mathrm{~s}$, delivery of $0.1 \mathrm{ml}$ of $15 \% \mathrm{EtOH}$ and a time-out of $2 \mathrm{~min}$, in which both levers were retracted. Rats were initially trained to press the seeking lever progressively under RI5-15-30-60 s, with increments occurring after 2 consecutive sessions under each RI schedule.

iv. Alcohol exposure: once performance on the seekingtaking chained schedule had stabilized ( $<10 \%$ betweensession variability), rats were divided into two subgroups in order to measure the influence of alcohol intake, under two different conditions, on subsequent behavioral performance: (i) group $1(n=47)$ underwent 8 sessions with only the taking lever available such that each taking lever response resulted in a delivery of $0.2 \mathrm{ml}$ of $15 \% \mathrm{EtOH}$. No limit was imposed on the number of reinforcements per $2 \mathrm{~h} / \mathrm{session}$ (instrumental exposure); (ii) Group $2(n=14)$ underwent 8 sessions with $4 \mathrm{~h}$ access to the $15 \% \mathrm{EtOH}$ bottle in the homecage. Alcohol intake $(\mathrm{g} / \mathrm{kg})$ was assessed for $4 \mathrm{~h}$ (onebottle exposure). Eight additional seeking-taking chained schedule sessions under RI60 were interspersed between either differential alcohol exposure sessions.

v. Seeking-taking punishment phase: each cycle began exactly as described for the seeking-taking phase. However, during each punishment session mild footshocks were randomly administered on completion of $30 \%$ of the cycles: the entire session consisted of 25 cycles, 17 reinforced by delivery of $0.1 \mathrm{ml}$ of $15 \% \mathrm{EtOH}$ following a taking lever response, whereas 8 were randomly punished by a $0.25-0.45 \mathrm{~mA}, 0.5 \mathrm{~s}$ foot-shock. The taking lever was never presented if a seeking cycle 
a General design of the experiment
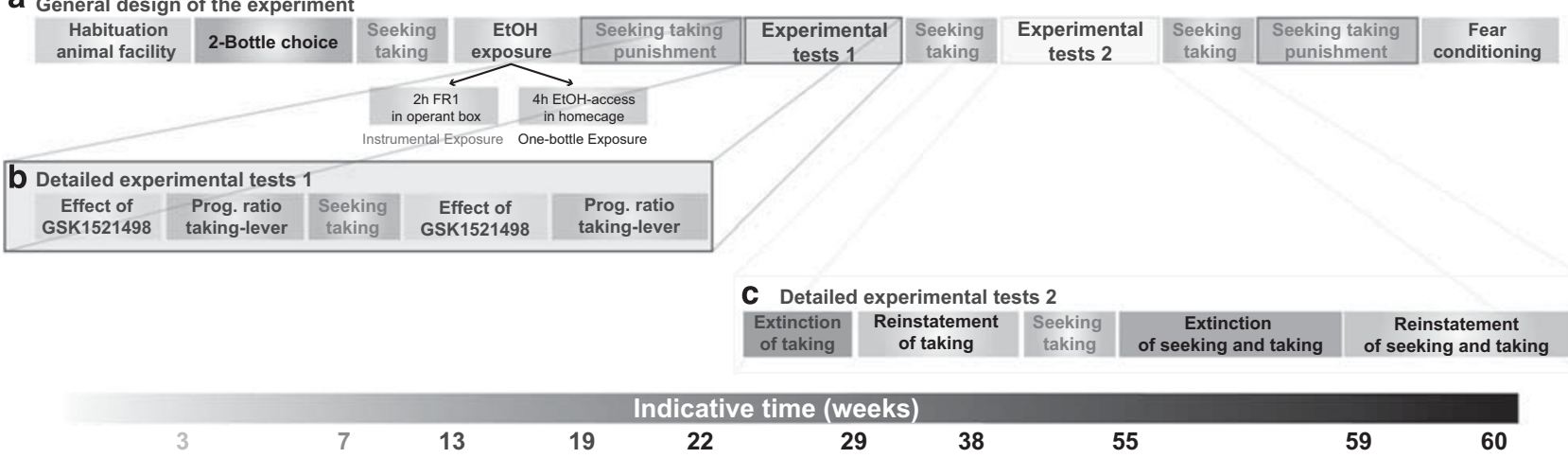

Figure I Timeline of the experiments carried out on the same cohort of alcohol-preferring rats. Experiment I was designed to establish a procedure to measure compulsive alcohol seeking behavior. (a) After a week of habituation to the animal facility and being pre-exposed to the intermittent 2-bottle choice procedure for 12 sessions, $P$ rats were trained to seek and take alcohol on a chained schedule of reinforcement. After being additionally exposed to alcohol access, either in the operant chamber under a fixed ratio I schedule ('Instrumental Exposure'), or in the home-cage ('One-bottle Exposure'), they were then trained under the seeking-taking chained schedule. After performance was stable, some seeking cycles randomly resulted in unpredictable mild foot-shock punishment, rather than insertion of the taking lever. At this stage rats were assigned to 'compulsivity-subgroups'. Rats then underwent two sets of experimental tests before being re-exposed ( 10 months after the last shock exposure) to the seeking-taking-punishment schedule. At the completion of the experiments, rats underwent fear conditioning to verify their sensitivity to shock in a Pavlovian conditioned fear procedure. (b) Once the compulsive nature of alcohol seeking was established according to the persistence of responding during punishment sessions, the effect of GSK I52 I 498 (I mg/kg, intraperitoneally) was measured on rats challenged under extinction of the seeking lever. Their motivation for alcohol was then tested under an exponential progressive ratio schedule on the taking lever only. After being re-baselined under the seeking-taking task (without shock exposure), the effect of GSKI52I498 under extinction, and the motivation for alcohol, were tested again in order to measure the influence of the expression of compulsivity on the effect of GSK I52 I 498 on alcohol seeking and the motivation of alcohol. (c) Rats were re-baselined under the seeking-taking task and underwent sessions of extinction of the taking lever followed by a session of cue-induced reinstatement of responding on the taking lever. After being re-baselined under the seeking-taking task, rats underwent a further 15 sessions of extinction of the seeking-taking chained response followed by a challenge session of cue-induced reinstatement of instrumental responding on both the seeking and taking levers.

resulted in punishment. Although the cycles were randomly punished during a session, the first cycle of the session was always reinforced and no more than 2 consecutive seeking cycles were punished. The intensity of the shock was progressively increased over daily sessions from 0.25 to $0.30,0.35,0.40 \mathrm{~mA}$, before stabilizing at $0.45 \mathrm{~mA}$ for 6 consecutive daily sessions. Ten months later, after completing several pharmacological and psychological manipulations (see experiments Figure 1), the same rats received an additional 6 sessions under the seeking-taking- $0.45 \mathrm{~mA}$ punishment schedule in order to investigate the stability of the compulsive alcohol seeking behavior phenotype over a protracted time period.

(ii) Fear conditioning. The rats tested for compulsive alcohol seeking were later tested for their sensitivity to shock using a fear conditioning procedure (Merlo et al, 2014). See SOM.

\section{Experiment 2: Influence of Systemic GSK1521498 Administration on Established Compulsive Seeking Behavior and Psychological Mechanisms Governing Established Compulsive Alcohol Seeking Behavior}

Experimental tests 1. Forty-eight hours after the last seeking-taking punishment session, rats received intraperitoneal injections of GSK1521498 1 mg/kg or vehicle, $30 \mathrm{~min}$ before a 15-min extinction session (alcohol was not available) the seeking lever only. On completion of the counterbalanced design, rats were challenged on the taking lever only, under an exponential progressive ratio (PR) schedule of reinforcement (PR). See SOM. The maximal number of responses that a rat performed to obtain $0.1 \mathrm{ml}$ of $15 \% \mathrm{EtOH}$ (the last ratio completed) was referred to as the break point, which provided a second measure of the motivation for alcohol.

Rats were then re-baselined under the seeking-taking (RI60-FR1) task and tested again being injected with GSK1521498 $0-1 \mathrm{mg} / \mathrm{kg}$ and under PR on the taking lever only under the same conditions described above, see Figure $1 \mathrm{~b}$.

Experimental tests 2. After extinguishing the taking lever responding, cue-induced reinstatement of instrumental responding on the taking lever was assessed in a 15-min test. During this test only the taking lever was present and each lever press resulted in a $20 \mathrm{~s}$-CS presentation only (no $\mathrm{EtOH}$ was delivered). Rats were then re-baselined under the seeking-taking (RI60-FR1) task. After extinguishing both the seeking and the taking lever responding, cue-induced reinstatement of both seeking and taking responses was assessed in a 15-min test. The session consisted of the seeking-taking (RI60-FR1) procedure as described before except for the absence of the alcohol delivery upon the taking lever press (only CS presentation, no EtOH delivery), see Figure 1c.

\section{Drugs}

See SOM. 


\section{Data and Statistical Analysis}

Data are presented as mean \pm SEM. Upon verification that assumptions concerning distribution and homogeneity of variance were not violated, data were subjected to repeated measures ANOVA with time or dose as within-subject factors and alcohol exposure condition and compulsivity as between-subject factors. Two-tailed values of $p \leqslant 0.05$ were considered statistically significant. Significant main effects and interactions were analyzed further using the Sidak's post hoc test where appropriate (SPSS 21, IBM, USA). Compulsive, intermediate and non-compulsive (NC) animals were identified using a two-step K-mean cluster analysis of the number of completed seeking-taking cycles during the last 3 days of the seeking-taking punishment schedule (footshock intensity $0.45 \mathrm{~mA}$; three data points per rat were considered). Cluster analysis has been shown to be a convenient and commonly used method for identifying objectively homogenous groups of objects within a data set (Tye et al, 2010; Murray et al, 2015; Engeln et al, 2016). Thus, the hypothesis-driven $\mathrm{k}$-means clustering with maximization of distance between groups defined a priori as 3 was performed on a unique dimension (compulsivity) but in a three-dimensional space (repeated measures). See SOM.

Spearman's rho correlational analyses were used to identify dimensional relationships between non-normally distributed populations pertaining to compulsivity (measured as the number of completed seeking-taking cycles under intermittent punishment) and: (i) motivation for alcohol (expressed by the breakpoints under PR schedule); (ii) seeking responses after extinguishing seeking-taking behavior; (iii) taking responses after extinguishing seekingtaking behavior; (iv) taking responses after extinguishing only taking behavior; and (v) GSK1521498 efficacy (measured as reduction of alcohol seeking) in C, I and NC rats were also computed. See SOM.

\section{RESULTS}

\section{Characterization of Compulsive Alcohol Seeking Behavior}

Since alcohol is a relatively weak reinforcer in most strains of rats (Spanagel and Hölter, 1999; Wolffgramm, 1991), we focused our attempt to establish a model of compulsive alcohol seeking on $\mathrm{P}$ rats, since they readily drink alcohol in excess of $5 \mathrm{~g}$ of alcohol/kg body weight/day and spontaneously prefer alcohol over water (McBride et al, 2014). They also display a marked propensity to seek instrumentally the opportunity to drink alcohol under the control of alcoholassociated conditioned stimuli (CSs), reaching blood alcohol concentrations of up to $80 \mathrm{mg} / \mathrm{dl}$ during $20 \mathrm{~min}$ of earned access (Giuliano et al, 2015).

Having established their alcohol preferring phenotype in an intermittent 2-bottle (alcohol/water) choice procedure, $\mathrm{P}$ rats were trained under a seeking-taking chained schedule (Belin-Rauscent et al, 2016; Chen et al, 2013; Pelloux et al, 2007; Figure 1, Supplementary Figure S1). Responding on one lever (seeking lever) under a RI $60 \mathrm{~s}$ schedule gave access to the opportunity to press a second lever (taking lever), responding on which under a FR 1 schedule resulted in delivery of $0.1 \mathrm{ml} 15 \%$ ethanol $(\mathrm{EtOH})$. $\mathrm{P}$ rats readily acquired, and maintained, high levels of alcohol seeking responses that were spatially and temporally independent of both alcohol taking responses and the opportunity to drink (Supplementary Figure S2). Upon introduction of unpredictable punishment of seeking responses (foot-shock intensity increasing from 0.25 to $0.45 \mathrm{~mA}$ over repeated sessions), approximately one-third of rats progressively decreased their alcohol seeking. By contrast, a subpopulation of rats maintained their seeking response despite punishment, thereby displaying compulsive alcohol seeking behavior (Figure 2a and b). A cluster analysis (Tye et al, 2010; Murray et al, 2015; Engeln et al, 2016) performed on the completed seeking-taking cycles during the last 3 sessions of punishment (Supplementary Figure S3) confirmed the existence of three subgroups of rats: $34 \%$ of the population displayed compulsive alcohol seeking (compulsive, C, rats, $n=21$ ), whereas, unexpectedly, a $30 \%$ sub-population greatly suppressed their seeking responses (NC rats, $n=18$ ); $36 \%$ of the population (intermediate, I, rats, $n=22$ ) between $\mathrm{C}$ and NC rats partially suppressed seeking when intermittently punished (compulsivity: $\mathrm{F}_{2,55}=84.55, p<0.001, \eta^{2}=0.75$ and time $\times$ compulsivity: $\left.\mathrm{F}_{18,495}=16.75, p<0.001, \eta^{2}=0.38\right)$. These data demonstrate that the propensity to drink alcohol is clearly not sufficient to predict the transition to compulsive alcohol seeking behavior (see SOM).

In people addicted to alcohol, the compulsive nature of alcohol seeking persists for months or even years, leading to relapse after even long periods of abstinence. Therefore, we investigated whether the compulsive alcohol-seeking phenotype in the vulnerable sub-population of $\mathrm{P}$ rats also persisted in the long term. We found that 10 months after initial characterization, the qualitative and quantitative nature of the compulsive behavior of these rats remained unchanged from its initial characterization (time: $\mathrm{F}_{1,32}<1$; shock session: $\mathrm{F}_{2,64}<1$; time $\times$ shock sessions: $\mathrm{F}_{2,64}=2.28$, ns; Figure 2c). This was not attributable to any difference in the aversiveness of the foot-shock, since all rats groups displayed the same conditioned fear response (Merlo et al, 2014 in addition to same appetitive conditioning, as demonstrated by Peña-Oliver et al, 2015) to a stimulus previously paired with shock of the same intensity as that used during the seeking-taking-shock task (conditioning: pre-CS vs CS1 and CS2: $\mathrm{F}_{2,44}=38.47, p<0.001, \eta^{2}=0.64$; compulsivity: $\mathrm{F}_{2,22}<1$; alcohol exposure: $\mathrm{F}_{1,22}<1$, conditioning $\times$ compulsivity: $\mathrm{F}_{4,22}<1$; conditioning $\times$ alcohol exposure: $\mathrm{F}_{2,22}<1$ ) (Figure 2d and e).

\section{Psychological Mechanisms Underlying Established Compulsive Alcohol Seeking Behavior}

We tested the potential contribution of motivational factors for alcohol of compulsive, intermediate and non-compulsive rats by measuring their performance on the lever associated with alcohol delivery (ie, taking lever) under a PR schedule of reinforcement (Richardson and Roberts, 1996), under two conditions: (i) in animals with a recent history of punishment (post-shock condition), thereby expressing their compulsive or NC phenotype; (ii) in animals re-trained under the seeking-taking task (post-baseline condition) so that performance under the PR was not influenced by punishment (Figure 3). Compulsive rats showed a higher 
motivation for alcohol than NC rats both prior to and after being exposed to punishment (compulsivity: $F_{2,41}=5.13$, $p=0.01, \quad \eta^{2}=0.20$; shock condition: $\left.\mathrm{F}_{1,41}<1\right)$, thereby suggesting that repeated exposure to punishment did not diminish the increased motivation for alcohol of compulsive rats. Although compulsive rats showed the same degree of a

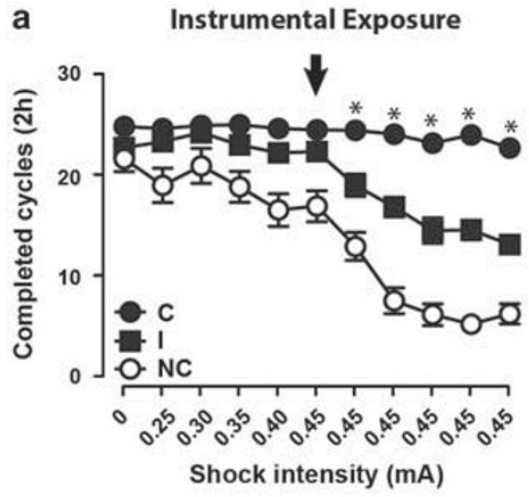

C

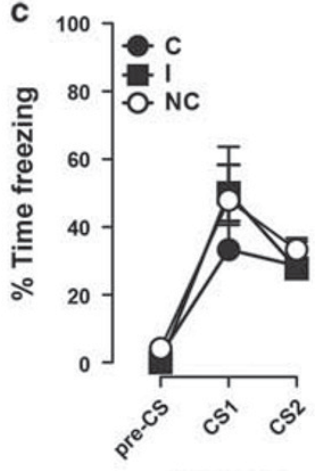

b

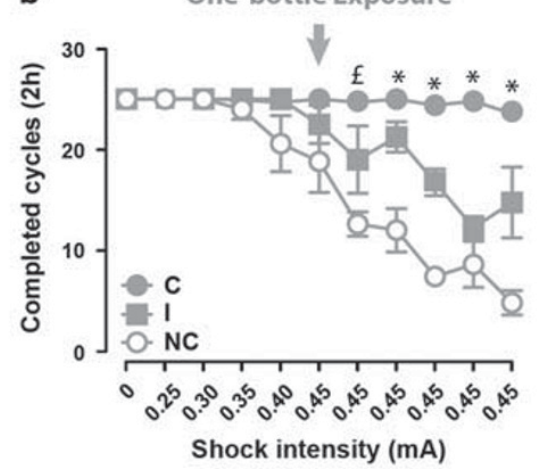

d

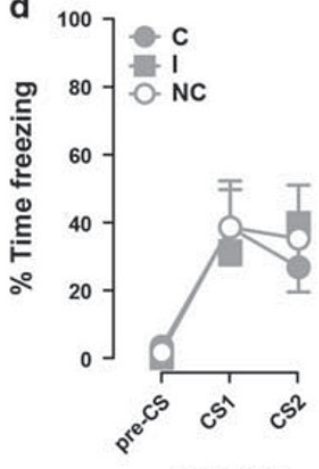

e $\quad 10$ Months Later

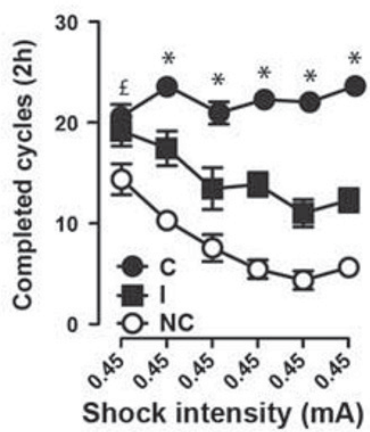

$\#<0.05$

$£<0.01$

${ }^{*}<0.001$

Figure 2 Compulsive alcohol seeking. (a and b) Alcohol-preferring rats were trained on a seeking-taking chained task to work for alcohol, and then their seeking responses were punished by mild electric foot-shocks of increasing intensity, from 0.25 to $0.30,0.35$, and $0.40 \mathrm{~mA}$, before stabilizing at $0.45 \mathrm{~mA}$ for six consecutive daily sessions. The arrow in each figure indicates the first session with a $0.45 \mathrm{~mA}$ foot-shock. During training, some had been given further exposure to alcohol either by working for it $(n=47)$ (Instrumental Exposure; A) or by being given free access to alcohol in the home-cage $(n=14)(O n e-$ bottle Exposure; B). Based on the persistence of alcohol seeking during the last three punishment sessions, measured as the number of completed seekingtaking cycles, a cluster analysis enabled the segregation of three subgroups of rats regardless of their previous history of alcohol exposure during training (Instrumental Exposure vs One-bottle Exposure): non-compulsive (NC, $n=18)$, intermediate $(I, n=22)$, and compulsive $(C, n=21)$ rats. $C$ rats completed more seeking-taking cycles/session than I and NC rats. The identified proportions were similar regardless of their specific alcohol exposure history (compulsivity-subgroups in Instrumental Exposure group vs compulsivity-subgroups in One-bottle Exposure group, Pearson $\chi^{2}=1.94$, ns). (c) Ten months after the last exposure to punishment, the same rats were again exposed to the seeking-taking shock task. Their compulsive alcohol seeking persisted and $C$ rats continued to complete significantly more seeking-taking cycles per session than I and NC rats, as revealed by the comparison between the last three punishment sessions at each time point. ( $\mathrm{d}$ and e) The differential resistance to punishment was not attributable to a difference in shock sensitivity. Following Pavlovian fear conditioning, rats showed similar levels of conditioned freezing in response to a CS previously paired with the identical shock to that used in the compulsive alcohol seeking task, regardless of their compulsivity or alcohol exposure history. ${ }^{\#} p<0.05 ;{ }_{p}<0.01$; ${ }^{*} p<0.001$ comparison of $C$ to $\mathrm{NC}$ rats.

Figure 3 Compulsive alcohol-preferring rats have a higher motivation for alcohol and reinstate alcohol seeking more readily than alcohol taking responses. (a and b) C rats $(n=14)$ had a higher motivation for alcohol than I $(n=17)$ or $N C(n=16)$ rats under a progressive ratio schedule as shown by a higher breakpoint (BP), on the left (white bars), and higher number of gained reinforcement per session, on the right (gray bars), measured either (i) after being trained under the seeking-taking punishment task (plain bars) or (ii) after being trained under baseline seeking-taking conditions (hatched bars), regardless of their previous history of shock condition. This difference was influenced by the alcohol exposure history (Instrumental Exposure (a) vs One-bottle Exposure (b)). (c and d) After 16 sessions of extinction in which only the taking lever was available and no alcohol was delivered, all rats eventually extinguished their taking responses (c). When presented with 20-s CSs contingent on taking lever responses (no alcohol was available at test), all rats reinstated their extinguished taking responses (cross-hatched bars) similarly over the 15-min period of the challenge, analyzed in 5-min-time-blocks, in particular during the first 5-min-timeblock of the session, prior to much extinction taking place within this test session. Rats $(n=33)$ reinstated their responding on the taking lever regardless of the compulsive nature of their behavior (d) (C rats $n=9$; I rats $n=13$; NC rats $n=\mid 1$ ). $* p<0.001$ differences from last day extinction responses (plain bars). (e-h) After 15 sessions during which both seeking and taking levers were presented under extinction conditions (no alcohol was delivered), all rats progressively reduced their alcohol seeking responses although $C$ rats $(n=9)$ maintained higher response rates compared to NC rats $(n=1 \mathrm{I})(e)$. However, they did not differ in their extinction of responding on the taking lever (g). During the 15-min cue-induced reinstatement test (cross-hatched bars), completion of the random interval (RI) $60 \mathrm{~s}$ of seeking-lever responses resulted in the availability of the taking lever, responding to which resulted in CS presentation, but not 
preference for alcohol over water as rats under the 2-bottle choice procedure, only rats that showed compulsive alcohol seeking behavior also showed a higher motivation for alcohol, thereby suggesting that this differential motivation for alcohol is dissociable from the propensity to drink alcohol. Yet, it was influenced by the alcohol exposure history as animals exposed to alcohol in their home-cages, after acquiring the seeking-taking task, had a higher baseline compared to animals exposed to alcohol during their instrumental training (alcohol exposure:
$\mathrm{F}_{1,41}=11.63, p<0.01, \eta^{2}=0.22$ and compulsivity $\times$ shock condition $\times$ alcohol exposure: $\mathrm{F}_{2,41}=4.29, p<0.05, \eta^{2}=0.17$; Sidak's post hoc: NC vs C, $p<0.05$; Figure $3 \mathrm{a}$ and $\mathrm{b}$ ).

One of the main consequences of developing compulsive alcohol seeking and drinking is a greater propensity to relapse during abstinence. Therefore, we further investigated the predictive validity of the model by measuring the propensity for alcohol-associated stimuli to trigger the reinstatement of both alcohol taking and alcohol seeking in compulsive, intermediate and rats after extinction. We
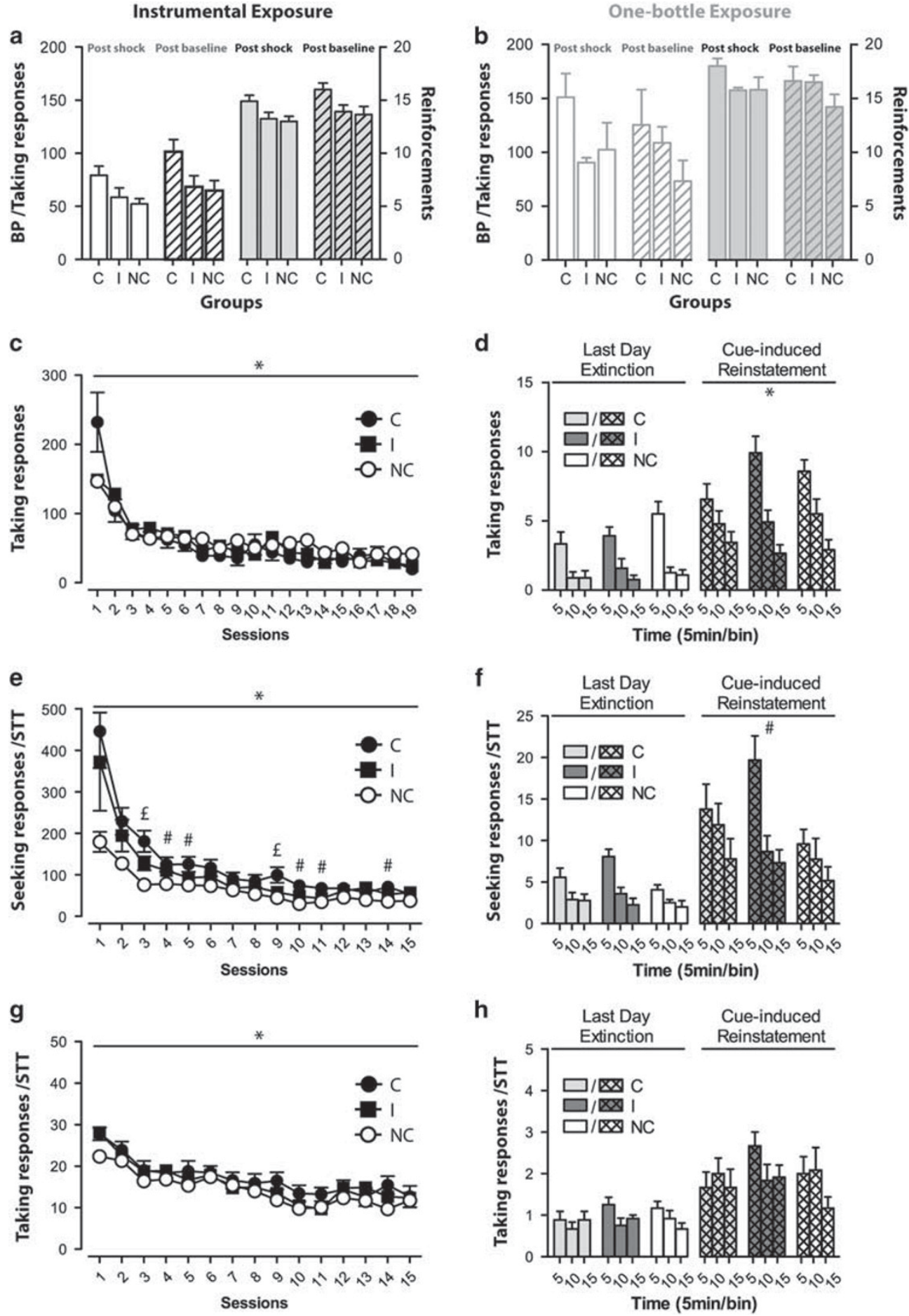

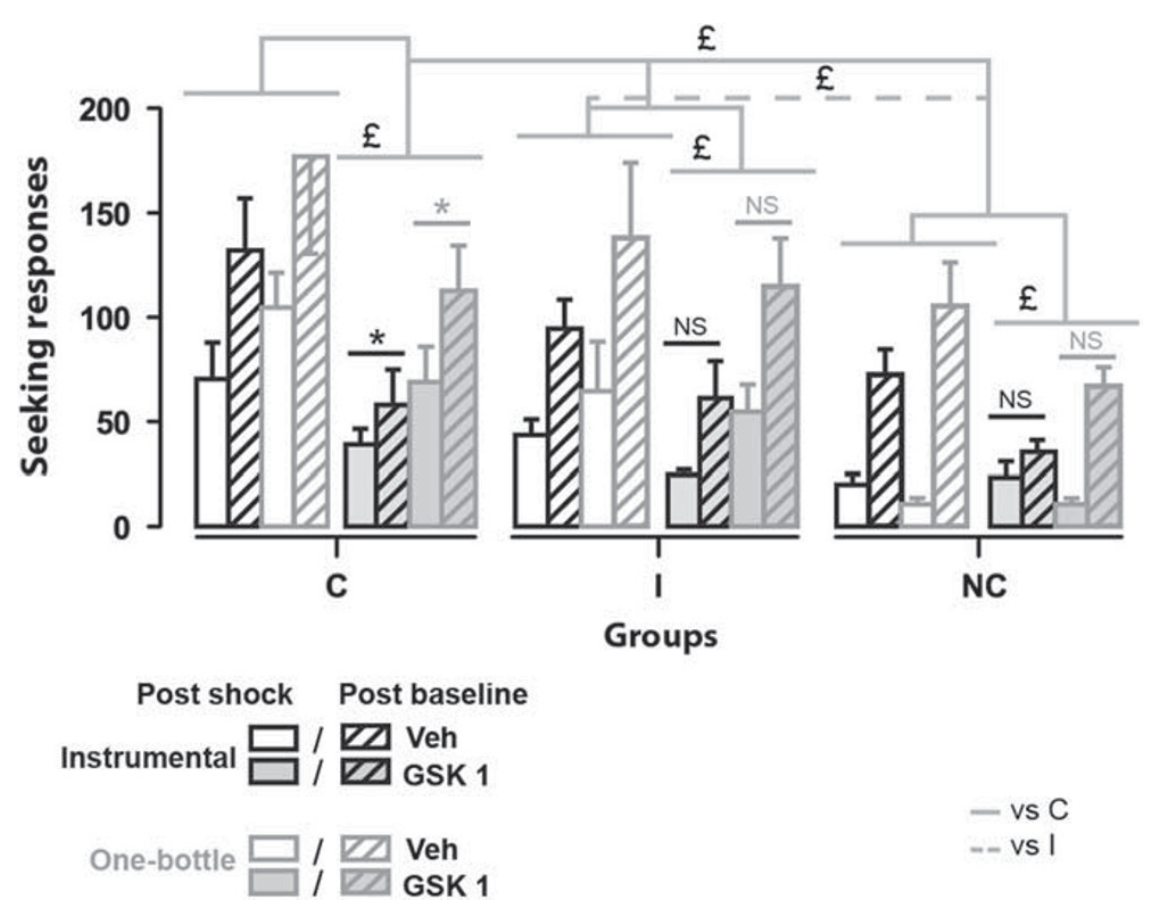

- vs C
-- vsl

Figure 4 GSKI521498 reduced seeking behavior in compulsive more than non-compulsive alcohol-preferring rats. The selective $\mu$-opiate receptor antagonist GSKI52I498 (I mg/kg, intraperitoneal) reduced alcohol seeking in rats with a history of alcohol exposure under Instrumental (black outlined bars) or One-bottle (gray outlined bars) exposure conditions during training and tested under a I5-min extinction challenge immediately following (i) a seekingtaking shock test session ('Post shock', plain bars) or (ii) a baseline seeking-taking test session ('Post baseline', hatched bars). The effect of GSKI52I498 in reducing alcohol seeking was more pronounced in C $(n=14)$ than NC $(n=16)$ rats. The decrease in alcohol seeking following GSKI52।498 administration was also influenced by the state under which rats were tested (Post shock, plain bars vs Post baseline, hatched bars) as rats tested 'Post baseline' had higher level of lever presses than rats trained 'Post shock', and the history of alcohol exposure (Instrumental Exposure, black bars vs One-bottle exposure, gray bars) as rats exposed to alcohol in their home-cage, after acquiring the seeking-taking task, had a general higher baseline compared to animals exposed to alcohol instrumentally during their operant training. Grey brackets show the main effects comparisons from C rats, whereas the dotted grey brackets show the main effect comparisons from I rats. Blue symbols indicate the comparison of GSK I 22 I 498-treated rats Instrumentally exposed to alcohol from their vehicle treated rats. Pink symbols indicate comparison of GSKI52 I498-treated rats One-bottle exposed to alcohol from their vehicle-treated rats. ${ }^{\#} p<0.05 ;{ }_{p}{ }_{p}<0.01$; * $p<0.00$ I comparison of GSKI521498 I mg/kg to vehicle treatment.

measured the propensity to relapse after: (i) selective extinction of the taking response (Figure 3c); (ii) extinction of the complete seeking-taking chain of responses (Figure 3e-g). In both cases, no alcohol or alcohol-associated CSs could be earned during the extinction phase, either by making taking responses or by completing seekingtaking cycles. In the first condition, while all rats eventually extinguished their instrumental responses on the taking lever (time: $\mathrm{F}_{15,450}=46.93, p<0.001, \eta^{2}=0.61$ ), compulsive rats displayed persistence of responding as compared to the other groups (time $\times$ compulsivity: $\mathrm{F}_{30,450}=2.84, p<0.01, \eta^{2}=0.16$; Figure 3c). Interestingly, in the second condition all rats progressively reduced their alcohol seeking responses (time: $\left.\mathrm{F}_{14,420}=29.46, p<0.001, \eta^{2}=0.50\right)$ although the compulsive rats maintained higher response rates compared with the noncompulsive (compulsivity: $\mathrm{F}_{2,30}=4.54, p<0.05, \eta^{2}=0.23$ and time $\times$ compulsivity: $F_{28,420}=1.80$, ns; Figure $3 \mathrm{e}$ ), but they did not differ in their extinction of responding on the taking lever (time: $\mathrm{F}_{14,420}=49.16, p<0.001, \eta^{2}=0.62$, compulsivity: $\mathrm{F}_{2,30}=$ 1.26, ns and time $\times$ compulsivity: $\mathrm{F}_{28,420}=1.16$, ns; Figure $3 \mathrm{~g}$ ). Reinstatement, a measure of relapse behavior, was assessed by reintroducing the alcohol-associated CS contingent on taking responses. Whereas all rats reinstated taking responses to similar levels (Figure 3d-h), intermediate and compulsive rats reinstated their seeking responses more than non-compulsive rats, thus indicating that only the relapse to seeking behavior is related to prior compulsivity (as detected by specifically analysing the seeking responses during the first $15 \mathrm{~min}$ of the last extinction session and the reinstatement session [reinstatement $\times$ compulsivity: $\left.\mathrm{F}_{2,30}=4.91, p<0.05, \eta^{2}=0.25\right]$ ) (Figure 3f).

\section{Systemic Administration of GSK1521498 Decreases Alcohol Seeking in Rats Identified as Compulsive}

We investigated the effect of systemic treatment with the $\mu$-opioid receptor antagonist GSK1521498 $(1 \mathrm{mg} / \mathrm{kg}$, intraperitoneally) on alcohol seeking behavior under two conditions: (i) immediately following punishment sessions, thereby testing animals while they expressed the compulsive nature of their seeking behavior (post-shock condition) or (ii) after training under baseline seeking-taking sessions, thereby testing animals actively seeking and consuming alcohol (post-baseline condition; Figure 4). The tests consisted of a 15-min session in which only the seeking lever was extended. GSK1521498 dramatically decreased alcohol seeking behavior under both conditions (treatment: $\left.\mathrm{F}_{1,41}=48.81, p<0.001, \eta^{2}=0.54\right)$ despite a lower level of responding following punishment as compared with baseline conditions (shock condition: $\mathrm{F}_{1,41}=39.96, \quad p<0.001$, $\eta^{2}=0.49$ ). The effect of GSK1521498 on alcohol seeking 
behavior was dependent upon compulsivity (compulsivity: $\mathrm{F}_{2,41}=10.17, p<0.001, \eta^{2}=0.33$, treatment $\times$ compulsivity: $\left.\mathrm{F}_{2,41}=6.30, \quad p<0.01, \eta^{2}=0.23\right)$, in that it was more pronounced in compulsive than NC rats.

\section{DISCUSSION}

\section{Compulsivity Develops in only a Subset of Vulnerable} Alcohol-Preferring P Rats

Taken together, the data reported here demonstrate that only a subset of vulnerable individuals develop compulsive alcohol seeking in a novel heuristic value and predictive validity that measures the persistence of this behavior in the face of adverse outcomes. In contrast to 'pharmacological-, or intoxication-centred' views of alcohol addiction (Koob, 2003; Koob et al, 1994), which suggest that the genetic determinants (whatever they may be in $\mathrm{P}$ rats, this is not the focus of the present study) of the individual propensity frequently to drink alcohol at levels high enough to result in intoxication may underlie alcohol addiction, the present study indicates that they are not necessary for the development of compulsive alcohol seeking. Our data show that a propensity to drink and spontaneously prefer alcohol is dissociable from the propensity compulsively to seek it, as well as that the transition to compulsivity in vulnerable rats was not predicted by their overall alcohol exposure prior to being exposed to punishment. Such a dissociation between the factors that predict an increased propensity to use, or self-administer drugs and those that instead predict the transition to compulsive drug seeking is not unprecedented. Previous studies demonstrated in alcohol-preferring $(\mathrm{P})$ rat lines that high alcohol intake does not necessarily lead to, or predict, the development of high levels of alcohol seeking behavior (Giuliano et al, 2015) or compulsive drinking (Vengeliene et al, 2009). This dissociation is also seen in experimental studies of cocaine addiction, whereby the propensity to self-administer stimulants by rats displaying a high response to novelty (HR) does not predict the vulnerability to develop compulsive cocaine seeking, which is instead predicted by high trait impulsivity (Belin et al, 2008). Indeed, HR rats are in fact resilient to the development of compulsive cocaine seeking (Belin et al, 2011). This suggests that the individual vulnerability to seek alcohol compulsively in rats with a high propensity to drink may depend upon environmental and epigenetic mechanisms (Russo et al, 2012), as also reported for cocaine (Vassoler et al, 2013), rather than on genetic polymorphism (Tabakoff et al, 2009). However, protracted alcohol exposure may facilitate a gradual transition in compulsivity-predisposed subjects, as demonstrated by the transition to punishment-resistant seeking after 10 months of alcohol exposure in some animals that had previously shown an intermediate phenotype. This vulnerability is also heterogeneous and, for some animals of the intermediate phenotype, more protracted exposure to alcohol was necessary to facilitate the transition to compulsivity. Thus, as revealed in the cue-induced reinstatement test which was conducted prior to the introduction of intermittent seeking punishment for the second time, a progression to compulsivity had occurred in a number of rats with the intermediate phenotype at some point during the 10-month period of alcohol exposure that followed the

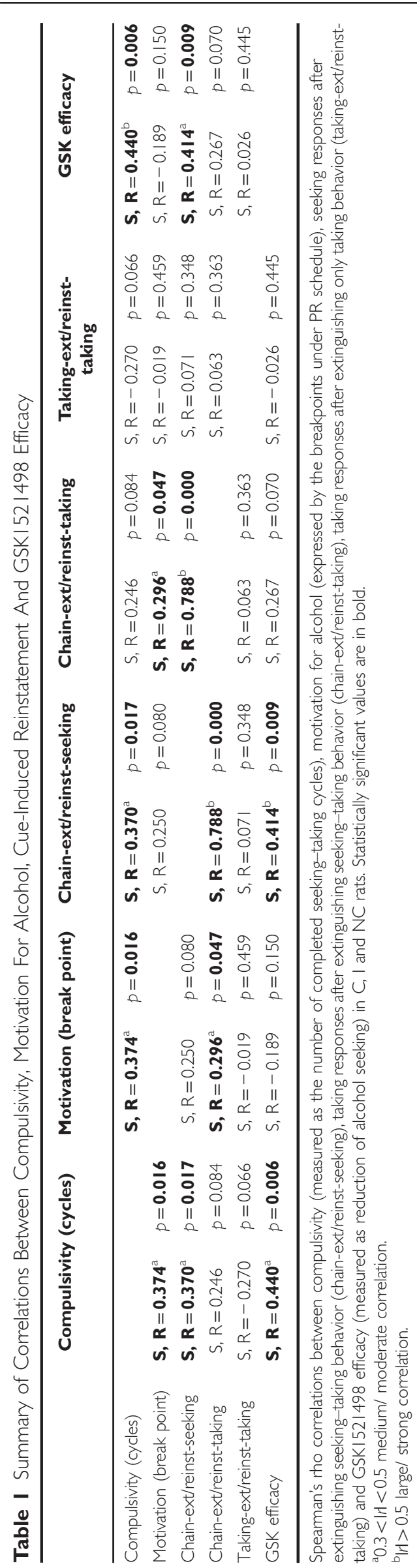


initial identification of inter-individual differences in the persistence of alcohol seeking despite punishment.

The persistence of responding in the face of punishment cannot be attributed to an anxiolytic effect of alcohol exposure in compulsive rats that, in maintaining high levels of seeking responding, gain more access to alcohol than noncompulsive rats (Barkley-Levenson and Crabbe, 2015). Thus, not only did rats receive only small intermittent volumes $(0.1 \mathrm{ml} /$ reward $)$ of alcohol on completion of each cycle of seeking and taking responses, but they all received a first rewarded cycle in each session in which punishment was then introduced probabilistically. Additionally, each rewarded and punished cycle is followed by a 2-min timeout, intended to minimize the short-term alcohol ingestion effects, and the carry-over effects of the physical properties of shock presentation, respectively.

\section{Compulsive Individuals may be more Prone to Relapse}

Compulsive and some intermediate individuals (whose compulsive phenotype only emerged after a much longer exposure to alcohol than their more vulnerable counterparts, thereby revealing some inter-individual differences in vulnerability to switch to compulsive alcohol seeking, as discussed above) were also more prone to relapse. In particular, compulsive alcohol seeking was shown to predict an increased propensity to reinstate seeking (see also Table 1 for correlation between compulsivity and relapse to seeking behavior), but not taking, responses following extinction, providing our model with further predictive validity. These data demonstrate that the compulsive nature of alcohol seeking predicts and translates into higher rates of relapse to alcohol seeking (but not taking) in compulsive rats, operationalized in the reinstatement procedure (Lê and Shaham, 2002), that is strongly influenced by alcoholassociated CSs, which are known to elicit craving and relapse in alcohol-dependent individuals (Epstein et al, 2006; Knight et al, 2016; Mayo and de Wit, 2016; O'Brien et al, 1992). Moreover identifying seeking responses as a key psychological component of compulsivity is in agreement with the evidence that seeking and taking (ie, preparatory and consummatory) responses, although clearly interrelated, are psychologically and neurally dissociable (Zapata et al, 2010).

\section{Mu-Opioid Receptor-Mediated Mechanisms Regulate Compulsive Alcohol Seeking Behavior}

Alcohol seeking responses were greatly decreased, especially in compulsive rats, by systemic administration of the selective $\mu$-opioid receptor antagonist GSK1521498 (see Table 1 for correlation between compulsivity and GSK1521498 reduction of seeking behavior). This was in addition to its effect of reducing both alcohol drinking and cue-controlled alcohol seeking reported in our earlier studies (Giuliano et al, 2015). Opioidergic mechanisms are thus involved in incentive motivational (anticipatory) responses for alcohol and the response-reinstating actions of ethanolassociated stimuli (Monti et al, 1999; Weiss et al, 2001), as well as the seeking of highly palatable food, cocaine and heroin (Czachowski and Delory, 2009; Giuliano et al, 2012, 2013, 2015; Ziauddeen et al, 2013). Opiate receptor antagonists inhibit the self-administration and intake of ethanol in a variety of animal models, suggesting that opioid pathways in the brain play a significant role in mediating the reinforcing properties of alcohol (Vengeliene et al, 2008), a view consistent with the clinical impact of approved medications for alcohol addiction, such as nalmefene and naltrexone which both reduce volumes of alcohol drunk. However, while reducing alcohol intake at initial relapse is of great value clinically, the marked propensity compulsively to seek drugs remains a major obstacle to attaining abstinence.

Here we present and validate a new behavioral procedure in rats that can be deployed to investigate the vulnerability to develop compulsive alcohol seeking, further showing that the novel selective $\mu$-opioid receptor antagonist GSK1521498 significantly reduces alcohol seeking in both compulsive and non-compulsive individuals. The accumulated data suggest that GSK1521498 may be a more generally effective $\mu$-opioid antagonist treatment across a broad spectrum of individuals addicted to alcohol, since it has the effect to decrease not only compulsive seeking behavior, but also the impact of alcohol cues and alcohol drinking that lead to relapse in otherwise abstinent individuals. Moreover, our findings are consonant with experimental medicine studies that have shown GSK1521498 to be well tolerated in humans and able to reduce self-reported responses to alcohol (Ziauddeen et al, 2016), indicating its significant translational potential as a potential treatment for alcoholism. Appropriately designed clinical trials of this putative treatment would be both indicated and timely.

The behavioral data in animals presented here recapitulate several psychological and behavioral aspects of human alcohol addiction that have not previously been operationalized in animal experimental procedures. Moreover, the alcohol seeking-taking punishment task can detect significant activity of a putative pharmacological treatment for alcohol addiction. The behavioral task we have developed will, we hope, greatly facilitate the experimental investigation of the cellular, molecular and neural circuit basis of alcohol addiction in a way that is directly relevant to the clinical disorder and its treatment.

\section{FUNDING AND DISCLOSURE}

The present study was funded by a Medical Research Council Programme Grant (no. G1002231) and by GlaxoSmithKline (GSK), which has a commercial interest in GSK1521498. The production of the $\mathrm{P}$ rats was funded by the R24 Alcohol Research Resource Award grant (R24 AA015512) from NIAAA. CG, YP-O, CRG, RNC, DB and BJE have no conflicting interests. TWR has consulted for GSK, Cambridge Cognition, Lilly, Lundbeck and Merck. He also has research grants with GSK, Lilly and Lundbeck. ETB is employed half-time by the University of Cambridge and halftime by GSK; he holds stock in GSK.

\section{ACKNOWLEDGMENTS}

We would like to thank Dr Larry Lumeng, Dr Richard Bell and Rebecca Jane Smith at the Indiana University School of Medicine for facilitating the provision of the selected lines of rats; Kristin Patterson and Ramprakash Govindarajan for 
providing the GSK1521498 solutions. CG, YP-O, DB and BJE designed the study and interpreted data; CG and YP-O performed experiments; CG and DB analyzed the data; TWR and BJE obtained funding and coordinated collaborations with Indiana University-Purdue University and with GlaxoSmithKline R\&D, Clinical Unit Cambridge; RNC provided the software program to perform the experiments; CG, DB and BJE wrote the manuscript; YP-O, CRG, RNC, TWR and ETB provided important conceptual inputs to the final version of the manuscript.

\section{REFERENCES}

American Psychiatric Association (2013). Diagnostic and Statistical Manual of Mental Disorders. Available at <http://dsm.psychia tryonline.org/doi/book/10.1176/appi.books.9780890425596>.

Barkley-Levenson AM, Crabbe JC (2015). Genotypic and sex differences in anxiety-like behavior and alcohol-induced anxiolysis in High Drinking in the Dark selected mice. Alcohol 49: 29-36.

Belin D, Berson N, Balado E, Piazza PV, Deroche-Gamonet V (2011). High-novelty-preference rats are predisposed to compulsive cocaine self-administration. Neuropsychopharmacology 36: 569-579.

Belin D, Mar AC, Dalley JW, Robbins TW, Everitt BJ (2008). High impulsivity predicts the switch to compulsive cocaine-taking. Science 320: 1352-1355.

Belin-Rauscent A, Fouyssac M, Bonci A, Belin D (2016). How preclinical models evolved to resemble the diagnostic criteria of drug addiction. Biol Psychiatry 79: 39-46.

Blasio A, Steardo L, Sabino V, Cottone P (2014). Opioid system in the medial prefrontal cortex mediates binge-like eating. Addict Biol 19: 652-662.

Carnicella S, Ron D, Barak S (2014). Intermittent ethanol access schedule in rats as a preclinical model of alcohol abuse. Alcohol 48: 243-252.

Chen BT, Yau H-J, Hatch C, Kusumoto-Yoshida I, Cho SL, Hopf FW et al (2013). Rescuing cocaine-induced prefrontal cortex hypoactivity prevents compulsive cocaine seeking. Nature 496: 359-362.

Cooper A, Barnea-Ygael N, Levy D, Shaham Y, Zangen A (2007). A conflict rat model of cue-induced relapse to cocaine seeking. Psychopharmacology (Berl) 194: 117-125.

Corbit LH, Nie H, Janak PH (2012). Habitual alcohol seeking: time course and the contribution of subregions of the dorsal striatum. Biol Psychiatry 72: 389-395.

Czachowski CL, Delory MJ (2009). Acamprosate and naltrexone treatment effects on ethanol and sucrose seeking and intake in ethanol-dependent and nondependent rats. Psychopharmacology (Berl) 204: 335-348.

Deroche-Gamonet V, Belin D, Piazza PV (2004). Evidence for addiction-like behavior in the rat. Science 305: 1014-1017.

Engeln M, Ansquer S, Dugast E, Bezard E, Belin D, Fernagut P-O (2016). Multi-facetted impulsivity following nigral degeneration and dopamine replacement therapy. Neuropharmacology 109: 69-77.

Epstein DH, Preston KL, Stewart J, Shaham Y (2006). Toward a model of drug relapse: an assessment of the validity of the reinstatement procedure. Psychopharmacology (Berl) 189: 1-16.

Everitt BJ, Robbins TW (2015). Drug addiction: updating actions to habits to compulsions ten years on. Annu Rev Psychol 67: 23-50.

Franck J, Jayaram-Lindström N (2013). Pharmacotherapy for alcohol dependence: status of current treatments. Curr Opin Neurobiol 23: 692-699.

Giuliano C, Goodlett CR, Economidou D, García-Pardo MP, Belin D, Robbins TW et al (2015). The novel $\mu$-opioid receptor antagonist GSK1521498 decreases both alcohol seeking and drinking: evidence from a new preclinical model of alcohol seeking. Neuropsychopharmacology 40: 2981-2992.

Giuliano C, Robbins TW, Nathan PJ, Bullmore ET, Everitt BJ (2012). Inhibition of opioid transmission at the $\mu$-opioid receptor prevents both food seeking and binge-like eating. Neuropsychopharmacology 37: 2643-2652.

Giuliano C, Robbins TW, Wille DR, Bullmore ET, Everitt BJ (2013). Attenuation of cocaine and heroin seeking by $\mu$-opioid receptor antagonism. Psychopharmacology (Berl) 227: 137-147.

Hendershot CS, Wardell JD, Samokhvalov AV, Rehm J (2016). Effects of naltrexone on alcohol self-administration and craving: meta-analysis of human laboratory studies. Addict Biol 14: (doi:10.1111/adb.12425).

Hopf FW, Lesscher HMB (2014). Rodent models for compulsive alcohol intake. Alcohol 48: 253-264.

Ignar DM, Goetz AS, Noble KN, Carballo LH, Stroup AE, Fisher JC et al (2011). Regulation of ingestive behaviors in the rat by GSK1521498, a novel micro-opioid receptor-selective inverse agonist. J Pharmacol Exp Ther 339: 24-34.

Kelly E, Mundell SJ, Sava A, Roth AL, Felici A, Maltby K et al (2015). The opioid receptor pharmacology of GSK1521498 compared to other ligands with differential effects on compulsive rewardrelated behaviours. Psychopharmacology (Berl) 232: 305-314.

Knight CP, Hauser SR, Deehan GA, Toalston JE, McBride WJ, Rodd ZA (2016). Oral conditioned cues can enhance or inhibit ethanol (EtOH)-seeking and EtOH-relapse drinking by alcohol-preferring (P) rats. Alcohol Clin Exp Res 40: 906-915.

Koob GF (2003). Alcoholism: allostasis and beyond. Alcohol Clin Exp Res 27: 232-243.

Koob GF, Rassnick S, Heinrichs S, Weiss F (1994). Alcohol, the reward system and dependence. EXS 71: 103-114.

Koob GF, Volkow ND (2010). Neurocircuitry of addiction. Neuropsychopharmacology 35: 217-238.

Lê A, Shaham Y (2002). Neurobiology of relapse to alcohol in rats. Pharmacol Ther 94: 137-156.

Marchant NJ, Khuc TN, Pickens CL, Bonci A, Shaham Y (2013). Context-induced relapse to alcohol seeking after punishment in a rat model. Biol Psychiatry 73: 256-262.

Mayo LM, de Wit H (2016). Acquisition of conditioned responses to a novel alcohol-paired cue in social drinkers. J Stud Alcohol Drugs 77: 317-326.

McBride WJ, Rodd ZA, Bell RL, Lumeng L, Li T-K (2014). The alcohol-preferring (P) and high-alcohol-drinking (HAD) ratsanimal models of alcoholism. Alcohol 48: 209-215.

McKim TH, Shnitko TA, Robinson DL, Boettiger CA (2016). Translational research on habit and alcohol. Curr Addict Rep 3: 37-49.

Merlo E, Milton AL, Goozée ZY, Theobald DE, Everitt BJ (2014). Reconsolidation and extinction are dissociable and mutually exclusive processes: behavioral and molecular evidence. J Neurosci 34: 2422-2431.

Monti PM, Rohsenow DJ, Hutchison KE, Swift RM, Mueller TI, Colby SM et al (1999). Naltrexone's effect on cue-elicited craving among alcoholics in treatment. Alcohol Clin Exp Res 23: 1386-1394.

Murray JE, Belin-Rauscent A, Simon M, Giuliano C, Benoit-Marand M, Everitt BJ et al (2015). Basolateral and central amygdala differentially recruit and maintain dorsolateral striatum-dependent cocaineseeking habits. Nat Commun 6: 10088.

O’Brien CP, Childress AR, McLellan AT, Ehrman R (1992). Classical conditioning in drug-dependent humans. Ann NY Acad Sci 654: 400-415.

Pelloux Y, Everitt BJ, Dickinson A (2007). Compulsive drug seeking by rats under punishment: effects of drug taking history. Psychopharmacology (Berl) 194: 127-137.

Peña-Oliver Y, Giuliano C, Economidou D, Goodlett CR, Robbins TW, Dalley JW et al (2015). Alcohol-preferring rats show goal 
oriented behaviour to food incentives but are neither signtrackers nor impulsive. PLOS ONE 10: e0131016.

Richardson NR, Roberts DC (1996). Progressive ratio schedules in drug self-administration studies in rats: a method to evaluate reinforcing efficacy. J Neurosci Methods 66: 1-11.

Ripley TL, Sanchez-Roige S, Bullmore ET, Mugnaini M, Maltby K, Miller SR et al (2015). The novel mu-opioid antagonist, GSK1521498, reduces ethanol consumption in C57BL/6J mice. Psychopharmacology (Berl) 232: 3431-3441.

Rösner S, Hackl-Herrwerth A, Leucht S, Vecchi S, Srisurapanont M, Soyka M (2010). Opioid antagonists for alcohol dependence. Cochrane Database Syst Rev 8: CD001867.

Russo SJ, Murrough JW, Han M-H, Charney DS, Nestler EJ (2012). Neurobiology of resilience. Nat Neurosci 15: 1475-1484.

Seif T, Chang S-J, Simms JA, Gibb SL, Dadgar J, Chen BT et al (2013). Cortical activation of accumbens hyperpolarization-active NMDARs mediates aversion-resistant alcohol intake. Nat Neurosci 16: 1094-1100.

Spanagel R (2000). Recent animal models of alcoholism. Alcohol Res Health 24: 124-131.

Spanagel R, Hölter SM (1999). Long-term alcohol self-administration with repeated alcohol deprivation phases: an animal model of alcoholism? Alcohol Alcohol 34: 231-243.

Tabakoff B, Saba L, Printz M, Flodman P, Hodgkinson C, Goldman D et al (2009). Genetical genomic determinants of alcohol consumption in rats and humans. BMC Biol 7: 70.

Turyabahika-Thyen K, Wolffgramm J (2006). Loss of flexibility in alcohol-taking rats: promoting factors. Eur Addict Res 12: 210-221.

Tye KM, Cone JJ, Schairer WW, Janak PH (2010). Amygdala neural encoding of the absence of reward during extinction. J Neurosci 30: $116-125$.
Vassoler FM, White SL, Schmidt HD, Sadri-Vakili G, Pierce RC (2013). Epigenetic inheritance of a cocaine-resistance phenotype. Nat Neurosci 16: 42-47.

Vendruscolo LF, Barbier E, Schlosburg JE, Misra KK, Whitfield TW, Logrip ML et al (2012). Corticosteroid-dependent plasticity mediates compulsive alcohol drinking in rats. J Neurosci 32: 7563-7571.

Vengeliene V, Bilbao A, Molander A, Spanagel R (2008). Neuropharmacology of alcohol addiction. Br J Pharmacol 154: 299-315.

Vengeliene V, Bilbao A, Spanagel R (2014). The alcohol deprivation effect model for studying relapse behavior: a comparison between rats and mice. Alcohol 48: 313-320.

Vengeliene V, Celerier E, Chaskiel L, Penzo F, Spanagel R (2009). Compulsive alcohol drinking in rodents. Addict Biol 14: 384-396.

Weiss F, Ciccocioppo R, Parsons LH, Katner S, Liu X, Zorrilla EP et al (2001). Compulsive drug-seeking behavior and relapse. Ann N Y Acad Sci 937: 1-26.

Wolffgramm J (1991). An ethopharmacological approach to the development of drug addiction. Neurosci Biobehav Rev 15: 515-519.

Zapata A, Minney VL, Shippenberg TS (2010). Shift from goaldirected to habitual cocaine seeking after prolonged experience in rats. J Neurosci 30: 15457-15463.

Ziauddeen H, Chamberlain SR, Nathan PJ, Koch A, Maltby K, Bush $M$ et al (2013). Effects of the mu-opioid receptor antagonist GSK1521498 on hedonic and consummatory eating behaviour: a proof of mechanism study in binge-eating obese subjects. Mol Psychiatry 18: 1287-1293.

Ziauddeen H, Nestor LJ, Subramaniam N, Dodds C, Nathan PJ, Miller SR et al (2016). Opioid antagonists and the A118G polymorphism in the $\mu$-opioid receptor gene: effects of GSK1521498 and naltrexone in healthy drinkers stratified by OPRM1 genotype. Neuropsychopharmacology 41: 2647-2657.

Supplementary Information accompanies the paper on the Neuropsychopharmacology website (http://www.nature.com/npp) 